\title{
Analysis of dissipation of a burst-type martensite transformation in a Fe-Mn alloy by internal friction measurements
}

\author{
P. C. W. Fung \\ Department of Physics and Centre of Materials Science, University of Hong Kong, Hong Kong \\ J. X. Zhang and Y. Lin* \\ Department of Physics, Zhongshan University, Guangzhou 510275, China \\ K. F. Liang \\ Department of Physics and Centre of Materials Science, University of Hong Kong, Hong Kong \\ and Department of Physics, Zhongshan University, Guangzhou 510275, China \\ Z. C. $\operatorname{Lin}^{\dagger}$ \\ Department of Physics, Zhongshan University, Guangzhou 510275, China
}

(Received 31 January 1996)

\begin{abstract}
Recently, we have proposed a theory to analyze the first-order phase transition (FOPT) in solids. In order to test the concept of the physics of dissipation during FOPT in solids, it is necessary to test the theory with different FOPT system. We study here a burst-type martensite transformation in a $\mathrm{Fe}-18.8 \% \mathrm{Mn}$ alloy sample for this purpose. We investigate the characteristics of $\gamma(\mathrm{fcc}) \rightleftharpoons \varepsilon(\mathrm{hcp})$ transformation in this alloy and measure the dependence of internal friction (IF) during $\gamma / \varepsilon$ transformation in varying rate of temperature $\dot{T}$ and vibration frequency $\omega$. For free oscillations, the IF was defined to be $Q_{\delta}^{-1}=\delta / \pi$ where $\delta$ is the logarithmic decrement. For general (forced) oscillations, IF is usually defined to be $Q_{w}^{-1}=(1 / 2 \pi)(\Delta W / W)$, where $\Delta W$ is the dissipation over one cycle, while $W$ is the maximum stored energy. During our analysis, the relation between $Q_{\delta}^{-1}$ and $Q_{w}^{-1}$ is deduced. The parameter $l$ (coupling factor between phase interface and oscillating stress) takes a small value (0.015-0.035) during PT, but takes a large value (0.86) during static state. The parameter $n$ (exponent of rate for effective PT driving force) takes a large value 0.33 during heating and 0.47 during cooling. The physical meaning of $n$ and $l$ is discussed. The methodology introduced here appears to be an effective way of studying FOPT in solids. [S0163-1829(96)02533-7]
\end{abstract}

\section{INTRODUCTION}

In the first paper of our series, a theory of dissipation function describing the first-order phase transformation (FOPT) in solids was presented. ${ }^{1}$ The low-frequency internal friction (IF) $Q^{-1}$ and the dissipation energy $\Delta G_{R}(T)$ describing the resistance against motion of phase interface (PI) during the FOPT covering a range of temperature in this theory can be expressed as ${ }^{1}$

$$
\begin{gathered}
Q^{-1}=B(T) \omega^{1-2 l}+A(T) \dot{T}^{n} / \omega^{n+2 l}, \\
\Delta G_{R}(T)=\frac{\Delta H}{T_{0}}\left(T-T_{0}\right)-A_{1}(T, \dot{T})\left(T-T_{S}\right)^{n}, \\
\Delta G_{R}(T) / \frac{\Delta H}{T_{0}}=\left(T-T_{0}\right) \\
\quad-k^{\prime} \frac{A(T)}{B(T)}\left(T-T_{S}\right)^{n} / \frac{\Delta H}{T_{0}},
\end{gathered}
$$

where $\omega$ is the measuring frequency of IF, $\dot{T}$ is the temperature varying rate, $n$ and $l$ are exponent factors, $\Delta H$ is the difference of enthalpy during FOPT; $k^{\prime}, A(T), B(T)$, and $A_{1}(\dot{T}, T)$ are parameters. We have shown mathematically in Ref. 1 that these parameters, such as $k^{\prime}, A(T), B(T)$,
$A_{1}(T, \dot{T})$, as well as the exponents $l$ and $n$ can be obtained from the IF measurements for different frequency $\omega$ and different temperature varying rate $\dot{T}$. The physical meaningful dissipation function $\Delta G_{R}(T)$ of FOPT in solids can therefore be calculated using Eq. (2) above.

To test the stated theory, we have demonstrated in Ref. 2 with a concrete example of a $\mathrm{VO}_{2}$ sample that indeed the dissipation energy $\Delta G_{R}(T)$ and the other relevant parameters can be found numerically. It is believed that in order to test further the theory of dissipation during FOPT, more FOPT systems with different characteristics should also be employed. We would note that in Ref. 2, during the FOPT, the $\mathrm{VO}_{2}$ ceramics changes its crystalline structure from monoclinic to tetragonal around $241 \mathrm{~K}$ with a diffusionless structural transformation. In this particular transformation, the thermal hysteresis $\Delta T=\left|T_{p}-T_{0}\right|$ is rather narrow, being about $4 \mathrm{~K}$, and the critical phase transformation driving force is small (less than $5 \mathrm{cal} / \mathrm{mol}$ ). The average velocity of the moving PI is relatively low during FOPT. In the Fe-18.8\% Mn alloy, one observes a FOPT with relatively fast speed in the propagation of the phase interface and a so-called diffusionless burst-type martensite transformation occurs. ${ }^{3-5} \mathrm{We}$ purposely choose such an alloy which possesses a large thermal hysteresis during $\varepsilon(\mathrm{hcp}) \rightleftharpoons \gamma(\mathrm{fcc})$ martensite PT, with $\Delta T=\left|T_{0}-T_{p}\right| \sim 50 \mathrm{~K}$, while a relatively high PT driving 
force $\Delta G_{d}$ is involved during the $\varepsilon / \gamma \mathrm{PT}$ (about $50 \mathrm{cal} / \mathrm{mol}$ ). On the other hand, the FOPT in $\mathrm{VO}_{2}$ is a reconstructional type during PT, and there is no crystallographic relation between the new phase and the parent phase. The martensite PT in the FeMn alloy, however, is a displacitive-type PT and there is a certain crystallographic orientation relation between the $\varepsilon$ phase and the $\gamma$ phase. ${ }^{3}$

As will be seen in Sec. III, the $\gamma / \varepsilon$ PT has a certain portion of isothermal PT (Ref. 3) and there is no modulus minimum during the $\gamma / \varepsilon$ PT. We have to test the dissipation theory more "directly" because we cannot get the relative modulus defect $\Delta \mu / \mu$ and calculate the $l$ value from the slope of $\ln \left(Q^{-1} / \Delta M / M\right)-\ln \left(\omega / \omega^{\prime}\right)$ (which is linear) as in the $\mathrm{VO}_{2}$ case. ${ }^{2}$ In this paper, we must fit the data of $Q^{-1}, \dot{T}$, and $\omega$ to our theoretical expressions directly and get the $l$ and $n$ values simultaneously. We believe it is a more rigorous test for our dissipation theory of FOPT.

We would remark that the martensite phase transformation (MPT) in the $\mathrm{Fe}-18.8 \% \mathrm{Mn}$ alloy is rather complicated $^{4-10}$ because there are three phases ( $\alpha$-bcc, $\gamma$-fcc, $\varepsilon$-hcp) involved during the PT in the temperature range from room $T(\mathrm{RT})$ to $523 \mathrm{~K}$. The fractions of residual $\gamma$ and $\alpha$ phases at RT and the fraction of residual $\alpha$ phase at $523 \mathrm{~K}$ are changing during thermal cycling. It is, therefore, necessary to train the sample in order to obtain a stable state with constant phase amount, at both the low-temperature (RT) side and the high-temperature $(523 \mathrm{~K})$ side. Another reason to use the Fe-Mn sample here is that the thermalexpansion coefficient of the $\gamma /(\alpha, \varepsilon)$ PT is remarkably large, being useful for a snap-type bimetal design, though there is still difficulty in controlling the transitions and stabilizing the completed phase.

On passing, we would note that as early as 1959 , Wang and co-workers ${ }^{8,9}$ measured the IF of a $\mathrm{Fe}-18 \% \mathrm{Mn}$ sample during both heating and cooling. They pointed out that (i) the peak height $Q_{p}^{-1}$ increases with increasing value of $(\dot{T} / \omega)$; (ii) a fairly high value of $Q^{-1}$ can be observed only if $\dot{T}$ $\neq 0$. If $Q^{-1}$ is measured suddenly at a certain temperature during PT under the situation where $\dot{T} \neq 0$, covering a temperature range until $\dot{T}=0$, the $Q^{-1}$ value will drop to the background value immediately. ${ }^{8,9} \mathrm{Ma}$ and Ke measured the same IF peak in $1964 .^{10}$ They changed the measuring temperature step by step, and a relatively shorter time was used (about $3 \mathrm{~min}$ ) to keep the temperature. They reported that the peak height increases slightly with increasing $\omega$. Wang and co-workers presented a theoretical model to explain the change in $Q^{-1}$ arising from the change of the elastic constant during the martensite transition. ${ }^{8,9,11,12} \mathrm{Ma}$ and Ke indicated that IF is associated with the motion of an extended dislocation at the coherent interface. ${ }^{10}$ Postnikov et al. considered that IF originates from the fluctuation of certain relevant quantities in the process of FOPT and derived an expression of IF which is proportional to $(\dot{T} / \omega) .{ }^{13}$ The implications of these early works will be discussed in Sec. V.

\section{SAMPLE PREPARATION AND EXPERIMENTATION}

Alloys were prepared from $99.9 \% \mathrm{Fe}, 99.9 \%$ Mn by melting in a high-frequency induction furnace under an argon atmosphere. After homogenization the ingots were hot- forged and then hot-rolled down to a thickness of $3 \mathrm{~mm}$. After removing oxide layers, the sheet was cut and drawn into a wire form with a diameter $1 \mathrm{~mm}$ and length $70 \mathrm{~mm}$. Chemical analysis showed that the alloy's ingredient has $18.8 \% \mathrm{Mn}$ by weight. The sample was finally annealed at $1000^{\circ} \mathrm{C}$ at vacuum under an axial stress for $10 \mathrm{~min}$, and then cooled down to room temperature at a rate of $25 \mathrm{~K} / \mathrm{min}$. Experimental investigations include dynamic and static measurements of the sample's internal friction (IF), modules, differential scanning calculation (DSC-2C), electricity resistance, and $\mathrm{x}$-ray diffraction $(\mathrm{D} / \mathrm{max}-3 \mathrm{~A})$ during heating and cooling processes.

Internal friction of the sample was measured by a vacuum inverted torsion pendulum (mode AITP-1, manufactured by Institute of Metal Research, Academic Sinica, Shengyang). The free decay mode was used for IF measurement with a maximum amplitude $1 \times 10^{-5}$. The sample was $50 \mathrm{~mm}$ long, with a diameter of $1.0 \mathrm{~mm}$.

IF measurements of the sample were carried out under the following conditions: (i) The sample was heated/cooled at a temperature rate of $\dot{T}=2{ }^{\circ} \mathrm{C} / \mathrm{min}$ for different measuring frequencies from $0.6-2 \mathrm{~Hz}$; (ii) the sample was free decay at a frequency around $f=2.0 \mathrm{~Hz}$, for different heating/cooling rate of $\dot{T}$ ranging from $0.5-3.5 \mathrm{~K} / \mathrm{min}$; (iii) for different frequencies, the sample was kept at constant temperatures (i.e., $\dot{T}=0$ ) in steps and $Q^{-1}$ was measured as a function of time. A built-in four-probe resistivity measurement device was used to collect the data of sample's resistance during transition, while IF and relative shear modulus were measured simultaneously. DSC data was also collected in order to define the driving force of transition. X-ray-diffraction analysis was carried out at various temperatures to check the appearance of the various phases during the complete thermal cycle.

According to the original definition of internal friction, theoretically $Q_{w}^{-1}=(1 / 2 \pi)(\Delta W / W)$ should be $<0.16$ in magnitude for the free decay mode, where $\Delta W$ and $W$ are the dissipated energy and the maximum stored elastic energy in a vibration cycle. During IF experimentation, the internal friction for free-decay-type oscillations is calculated according to $Q_{\delta}^{-1}=\delta / \pi=(1 / n \pi) \ln \left(A_{0} / A_{n}\right)$, where $n$ is the number of cycles and $A_{0}, A_{n}$ are the corresponding amplitudes. When $Q^{-1}$ is small (e.g., $Q^{-1} \sim 0.02$ ), the above two expressions are practically equal. When IF is relatively large (e.g., $\left.Q^{-1} \gtrsim 0.05\right), Q_{\delta}^{-1}$ can be significantly different from $Q_{w}^{-1}\left(Q_{\delta}^{-1}>Q_{w}^{-1}\right)$. In the measurements involved in this paper, the IF can be as large as 0.3 and $Q_{\delta}^{-1}$ does not represent the dissipation in one cycle of oscillation. Note that as the IF measured using FOPT is independent on the vibration amplitude $A_{\varepsilon}$ in the $A_{\varepsilon}$ range used here $\left(\sim 10^{-5}\right), W \propto A_{n}^{2}$ we have

$$
\begin{aligned}
Q_{w}^{-1} & =\frac{1}{2 \pi} \frac{\Delta W}{W}=\frac{1}{2 \pi} \frac{A_{n}^{2}-A_{n+1}^{2}}{A_{n}^{2}}=\frac{1}{2 \pi}\left(1-e^{-2 \delta}\right) \\
& =\frac{1}{2 \pi}\left[1-\exp \left(-2 \pi Q_{\delta}^{-1}\right)\right] .
\end{aligned}
$$

While experimentally we measure $Q_{\delta}^{-1}$, we use $Q_{w}^{-1}$ in our theoretical deduction. The influence of the difference be- 

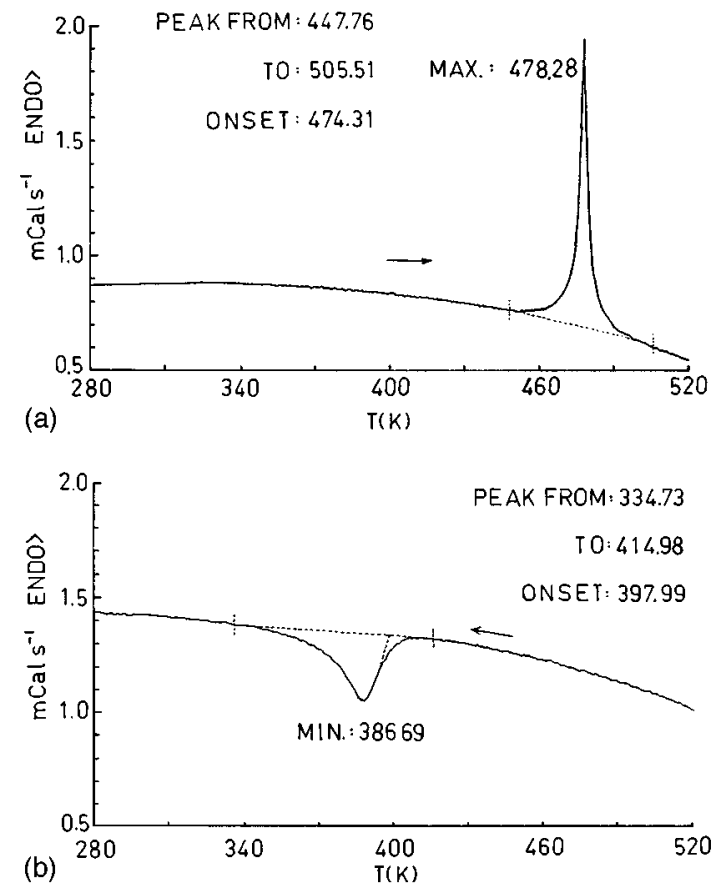

FIG. 1. DSC curves of FeMn sample with $\dot{T}=5 \mathrm{~K} / \mathrm{min}$. (a) during heating, (b) during cooling.

tween $Q_{w}^{-1}$ and $Q_{\delta}^{-1}$ on the values of $n$ and $l$ will be discussed in Sec. V.

\section{EXPERIMENTAL RESULTS}

In order to confirm the physical nature of the FOPT in the Fe-18 wt. \% Mn sample, DSC, thermal-expansion coefficient, electrical resistance, shear modulus, and internal friction $Q^{-1}$ (IF) measurements were carried out. The DSC curves during heating and cooling are shown in Fig. 1. We observe clearly that a typical DSC peak of FOPT appears at 478.3 $\mathrm{K}$ during heating and at $386.7 \mathrm{~K}$ during cooling in the FeMn sample. In Fig. 2, the IF $Q_{\delta}^{-1}$ (a), relative shear modulus $\left(f / f_{0}\right)^{2}(\mathrm{~b})$, electric resistance $\Delta R(\mathrm{c})$, and relative expansion coefficient $\Delta L / L_{0}$ (d) verses $T$ during a thermal cycle are shown. It is apparent that the thermal hysteresis $\Delta T=\left|T_{0}-T_{p}\right|$ has a large value $(\gtrsim 50 \mathrm{~K})$ for the $\gamma / \varepsilon$ bursttype MPT. During heating $(\varepsilon \rightarrow \gamma)$, a high IF peak corresponds to a large decrease of shear modulus and to an increase of electrical resistance $\Delta R$ as well as thermalexpansion coefficient rate $\Delta L / L$. During cooling $(\gamma \rightarrow \varepsilon)$, a lower but broader IF peak corresponds to a large increase of shear modulus and to a decrease of $\Delta R$ as well as expansion rate $\Delta L / L$. The abnormal variation of the shear modulus around $355 \mathrm{~K}$ is associated with the antiferromagnetic-toparamagnetic transition in the residual $\gamma$ phase. ${ }^{14}$ This is a second-order PT and there is, therefore, no thermal hysteresis as well as no interaction between the low-frequency vibrating stress and the magnetic transition. Figure 3 shows the $\mathrm{x}$-ray-diffraction patterns, which demonstrate the variation of $\gamma, \alpha$, and $\varepsilon$ phases during the first thermal cycle. It is noted that after one thermal cycle, the residual $\gamma$ phase and $\alpha$ phase increase at $303 \mathrm{~K}$. The variation of $Q_{\delta}^{-1}-T$ curves during the first, fourth, and fifth thermal cycle are indicated
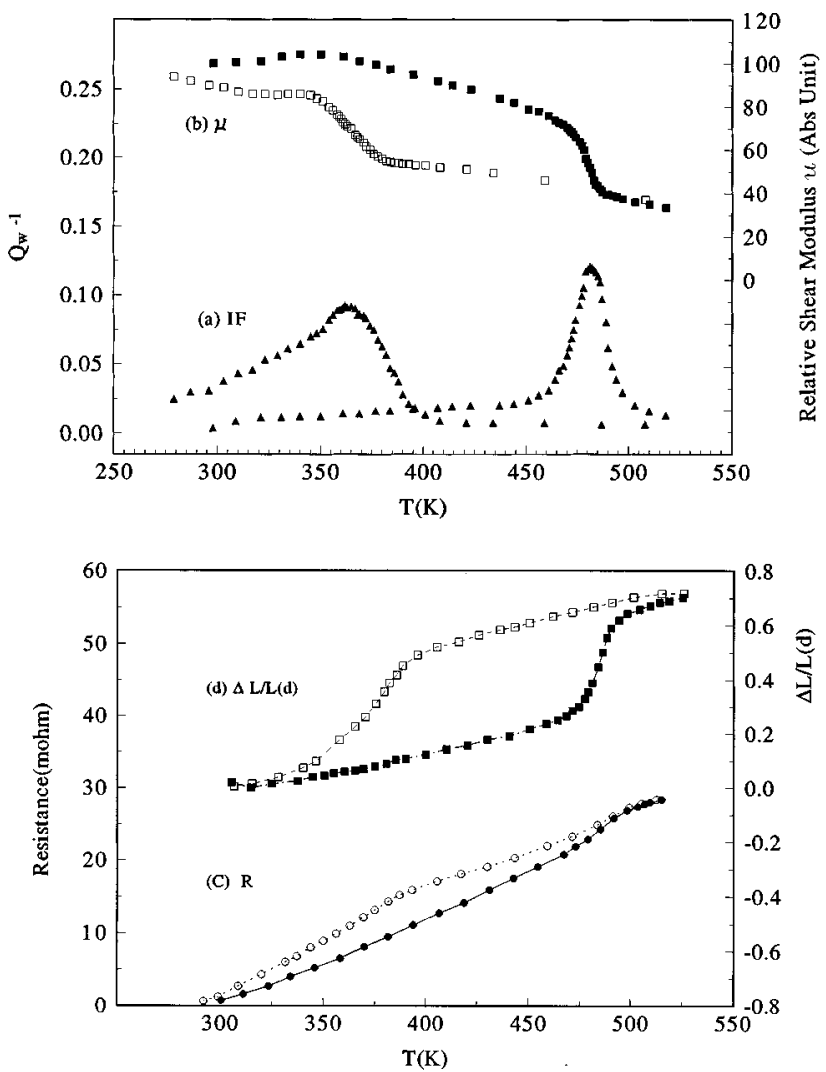

FIG. 2. Internal friction (IF) $Q_{w}^{-1}$ (a) and relative shear modulus $\mu$ (b) during heating and cooling at $f=1.0 \mathrm{~Hz}$ and $\dot{T}=2.0 \mathrm{~K} / \mathrm{min}$. Electrical resistance $R$ (c) and thermal-expansion coefficient $\Delta L / L$ (d) versus temperature at $\dot{T}=2.0 \mathrm{~K} / \mathrm{min}$.

in Fig. 4. After the sample has gone through a series of thermal cycles, the phase content, as indicated by the phase ratios of the $\gamma, \varepsilon$, and $\alpha$ phases at the low-temperature side (around $303 \mathrm{~K}$ ) and at the high-temperature (around $523 \mathrm{~K}$ ) tend to a stable state. The values of the amplitude of $Q^{-1}$ peak (i.e., $Q_{p}^{-1}$ ) and the thermal hysteresis $\Delta T=\left|T_{0}-T_{p}\right|$ as well as $\Delta G_{d}$ are plotted against the number of thermal cycles $N$ in Fig. 5. We observe from Fig. 5 that after the sixth thermal cycle, all the stated three quantities tend to constant values.

Figure 6(a) shows the variations of $Q_{\delta}^{-1}$ and relative shear modulus $\mu$ with respect to change in temperature for four different temperature varying rates $\dot{T}$ as marked and at a constant measuring frequency $f=1 \mathrm{~Hz}$. For three measuring frequencies as marked and at a constant temperature rate $\dot{T}=2.0 \mathrm{~K} / \mathrm{min}$, we have $Q_{\delta}^{-1}-T$ and $\mu-T$ plots as shown in Fig. 6(b). Note that an IF peak appears during both heating and cooling and that the peak height $Q_{p}^{-1}$ increases with increasing $\dot{T}$ but decreases with increasing frequency. The IF peaks during heating are higher than those of the cooling peaks, but the peak form of a cooling peak is broader than that of heating one in general. There is no minimum in the $\mu-T$ curve, but the relative modulus of the $\varepsilon$ phase is much higher than that of $\gamma$ phase. From the $\mu-T$ curve, we cannot find the minimum of the modulus defect; therefore, the $l$ value cannot be calculated from the modulus defect. Using the data in Figs. 6(a) and 6(b), we deduce the $Q_{w}^{-1}-T$ rela- 

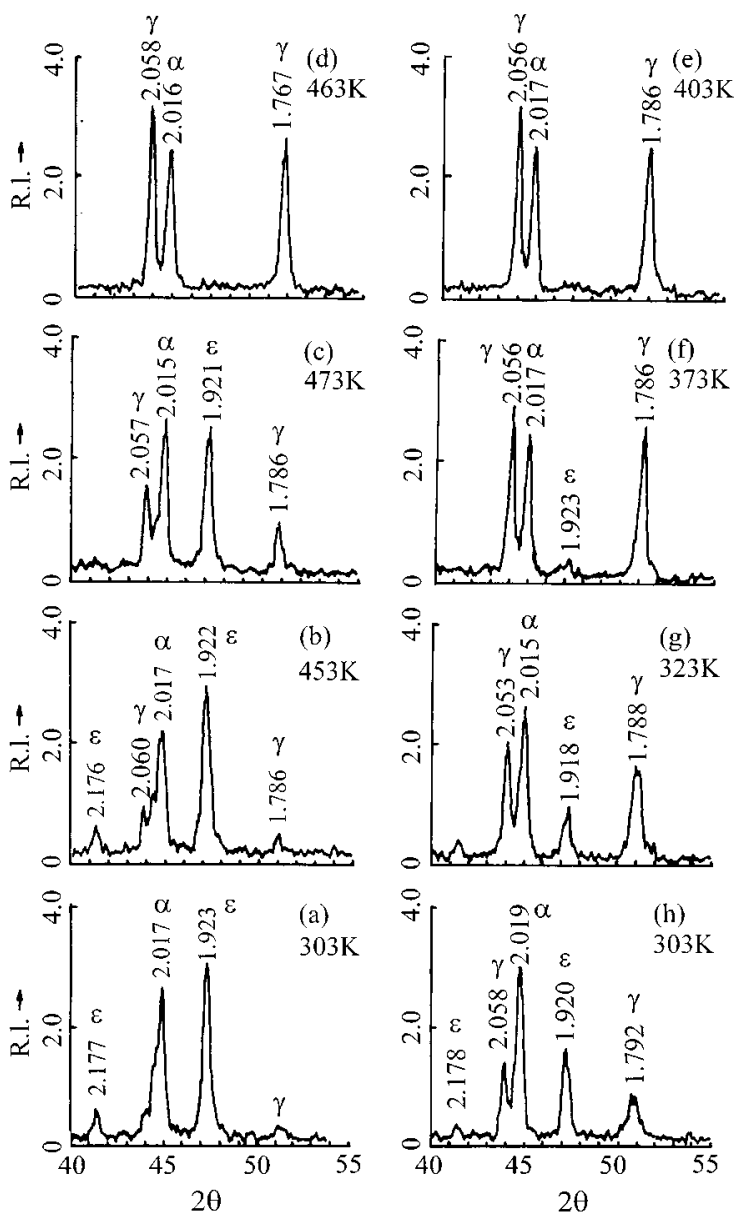

FIG. 3. X-ray-diffraction spectrum (relative intensity R.I. versus $2 \theta)$ at various temperatures during a thermal cycle. The sample was heated from (a) $303 \mathrm{~K}$ to (b) $453 \mathrm{~K}$ then to (c) $473 \mathrm{~K}$. When the temperature $523 \mathrm{~K}$ was reached, the sample was cooled down to (d) $463 \mathrm{~K}$. The sample was then cooled further down to (e) $403 \mathrm{~K}$, (f) $373 \mathrm{~K}$, (g) $323 \mathrm{~K}$, and (h) $303 \mathrm{~K}$.

tions according to Eq. (3) and present such plots in Figs. 7(a) and 7(b). Comparing Figs. 6 and 7, we note that the maximum value of $Q_{\delta}^{-1}$ is equal to 0.30 in Fig. 6, while that of $Q_{w}^{-1}$ is only 0.135 . The subscript $w$ will be omitted hereafter.

The sample was heated to $482 \mathrm{~K}$ (which is just the $T_{p}$ value during heating) and IF was measured at this temperature with aging time $t_{a}$, at a frequency of $2.0 \mathrm{~Hz}$. Such a $Q^{-1}-t_{a}$ result is shown in Fig. 8. The steady $Q^{-1}$ value after $t_{a} \approx 50 \mathrm{~min}$ of Fig. 8 becomes one datum point of Fig. 9. In other words, each point in Fig. 9 is the $Q^{-1}$ value for a particular $T$ after a sufficient aging time when $Q^{-1}$ becomes stabilized. Each curve in Fig. 9 pertains to a particular frequency during either heating or cooling as marked. It is clear from comparing Figs. 7 and 9, that the contribution of the dynamic term $A(T) \dot{T}^{n} / \omega^{n+2 l}$ [in Eq. (1)] to the IF is at least two orders of magnitude larger than that of the static one and the value of $A(T)$ is also at least $10^{3}$ times larger than that of parameter $B$.

\section{DATA ANALYSIS AND THE CHARACTERIZATION OF THE DISSIPATION FUNCTION $\Delta G_{R}$}

Having recorded the relevant data based on experimentation, we are in a position to analyze such data, with the aim

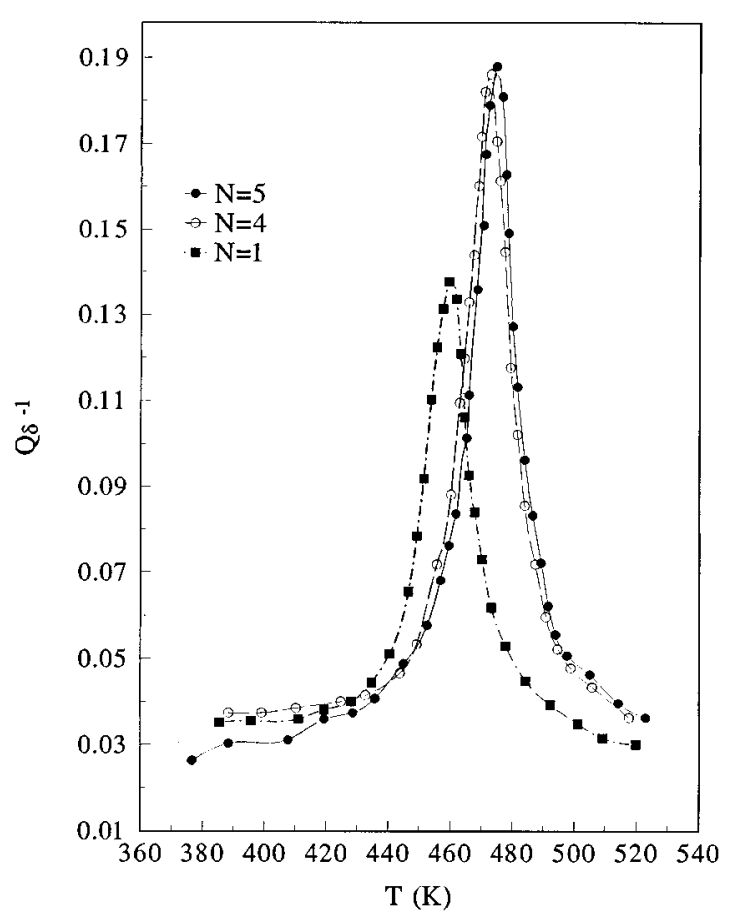

FIG. 4. Variation of internal friction during the first, fourth and fifth cycle.

to characterize the dissipation function and consequently to provide physical meanings for the parameters $l, n$ of this sample.

Based on data points in Fig. 7(a), we can find the peak height $Q_{p}^{-1}$ for various $\dot{T}$ at a practically constant $T$ and at

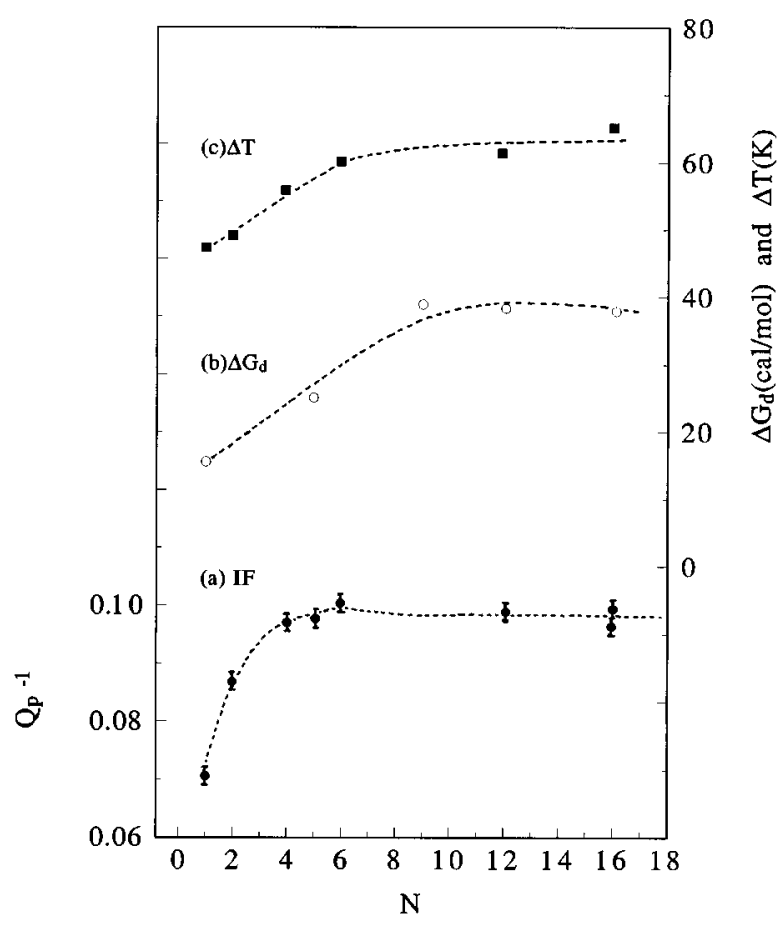

FIG. 5. Variation of IF with $\dot{T}=2.0 \mathrm{~K} / \mathrm{min}$ (a), PT driving force $\Delta G_{d}$ with $\dot{T}=5 \mathrm{~K} / \mathrm{min}$ (b) and thermal hysteresis $\Delta T=\left(T_{0}-T_{p}\right)$ with $\dot{T}=2.0 \mathrm{~K} / \mathrm{min}$ (c) versus the number of thermal cycle. 

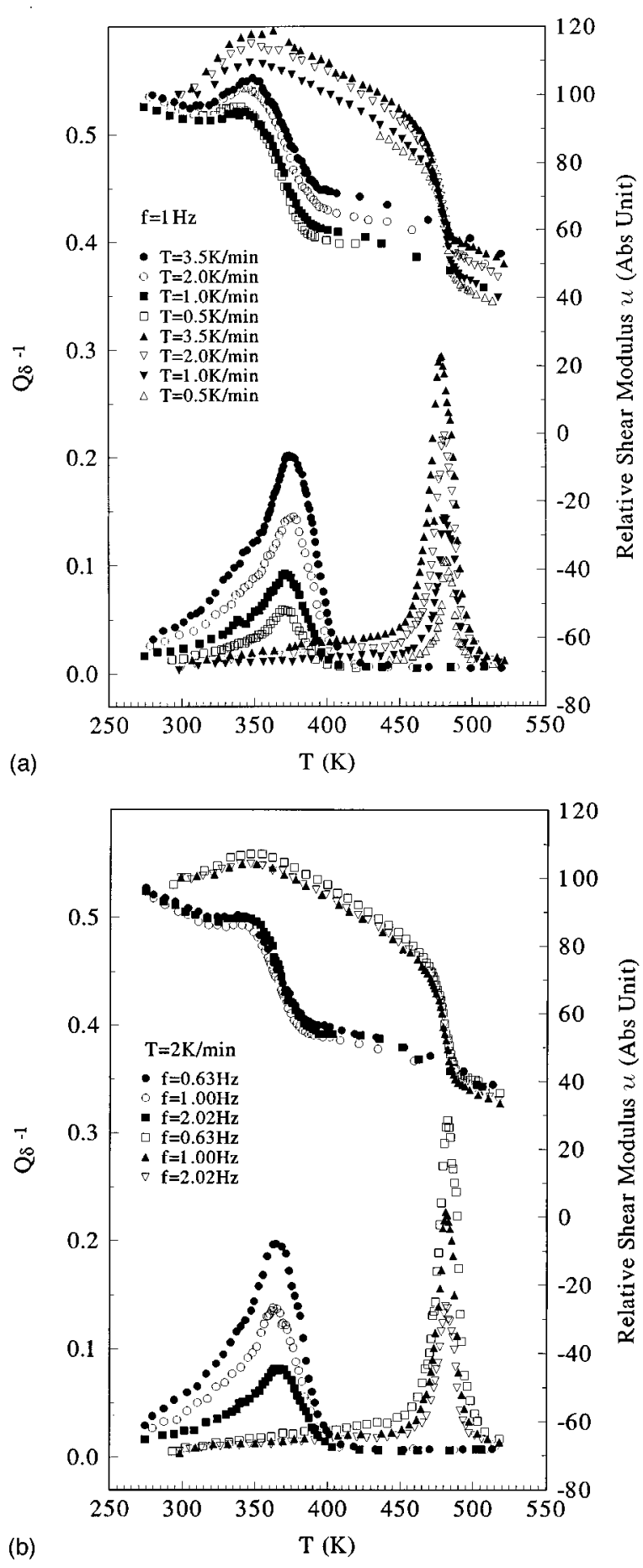

FIG. 6. (a) $Q_{\delta}^{-1}$ and relative shear modulus $\mu$ versus $T$ (K) for four temperature varying rates $\dot{T}$ as marked, at a frequency of $f=1 \mathrm{~Hz}$. (b) $Q_{\delta}^{-1}$ and relative shear modulus $\mu$ versus $T(\mathrm{~K})$ for three values of frequency $f$ as marked, while $\dot{T}=2.0 \mathrm{~K} / \mathrm{min}$ is constant.

constant frequency of $1.0 \mathrm{~Hz}$. We can therefore plot $\ln$ $Q_{p}^{-1}-\ln \dot{T}$ in Fig. 10 during heating as well as cooling. Since both lines appear linear, we can express $Q_{p}^{-1}$ as

$$
Q_{p}^{-1} \propto \dot{T}^{n^{\prime}},
$$
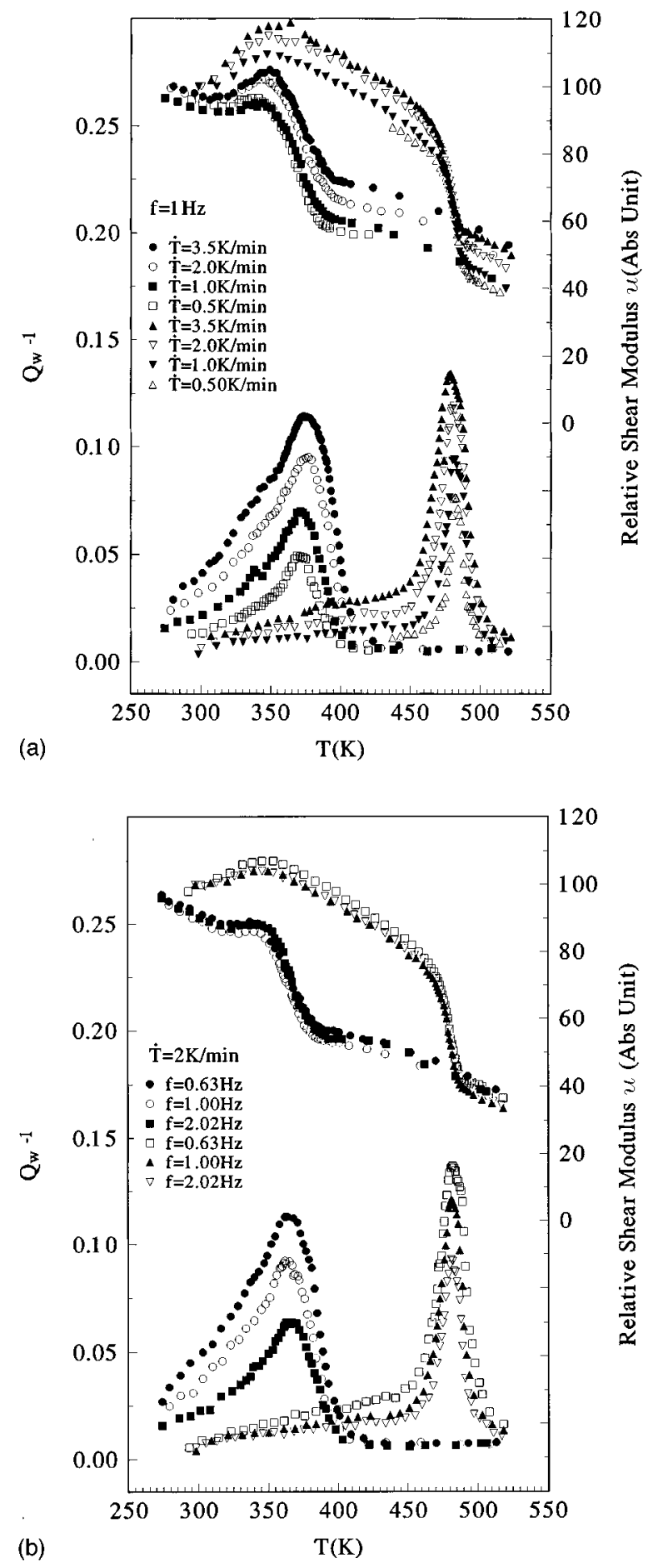

FIG. 7. (a) $Q_{w}^{-1}$ and relative shear modulus $\mu$ versus $T$ (K) for four temperature varying rates $\dot{T}$ as marked, at a frequency of $f=1 \mathrm{~Hz}$. (b) $Q_{w}^{-1}$ and relative shear modulus $\mu$ versus $T$ (K) for three values of frequency $f$ as marked, while $\dot{T}=2.0 \mathrm{~K} / \mathrm{min}$ is constant.

the parameter $n^{\prime}$ for the heating process is found to be 0.33 , while that for the cooling process is 0.44 . Likewise, based on data of Fig. 7(b), we can show (in Fig. 11) the variation of ln $Q_{p}^{-1}$ with respect to change in $\ln f$, while $\dot{T}=2 \mathrm{~K} / \mathrm{min}$, being constant. Again, linearity of the lines suggests that we can write 


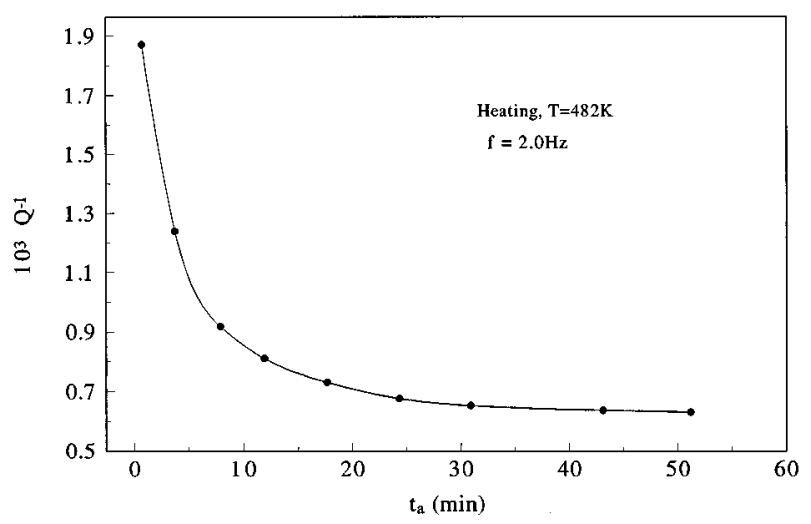

FIG. 8. Internal friction $Q^{-1}$ against aging time $t_{a}$ at $T=428 \mathrm{~K}$ after heating, operated at frequency of $2.0 \mathrm{~Hz}$.

$$
Q_{p}^{-1} \propto f^{-n^{\prime \prime}}
$$

and the values of $n^{\prime \prime}$ found during heating and cooling are, respectively, 0.34 and 0.53 .

In view of Eqs. (4) and (5), $Q_{p}^{-1} \propto \dot{T}^{n^{\prime}} / f^{n^{\prime \prime}}$, equating $Q_{p}^{-1}$ to the dynamic term of $Q^{-1}$ in Eq. (1), we see at once that $l=1 / 2\left(n^{\prime \prime}-n^{\prime}\right)$. Putting in values of $n^{\prime}, n^{\prime \prime}$ from Figs. 10 and 11 , we observe that for both heating and cooling processes, $l \approx 0.005$ and 0.045 , respectively. According to earlier work reported in Refs. 8 and $9, Q_{p}^{-1}$ was assumed to be expressible explicitly as $Q_{p}^{-1} \propto(\dot{T} / f)^{n}$. We see that such an assumption represents roughly an average of Figs. 10 and 11. We can in fact test this idea by plotting $\ln Q_{p}^{-1}$ versus $\ln (\dot{T} / f)$. The slope found during heating is $n=0.32$ [while $\left.\left(n^{\prime \prime}+n^{\prime}\right) / 2=0.335\right]$ and that during cooling is $n=0.47$ [while $\left(n^{\prime \prime}+n^{\prime}\right) / 2=0.485$ ]. We must note that the data points follow the straight lines very closely in Figs. 9 and 10, while the points are relatively scattered in Fig. 12. The other parameter $l$ would not appear in the relation $Q_{p}^{-1} \propto(\dot{T} / f)^{n}$, meaning that $l$ is assumed to be zero in Eq. (1). However, we have shown in Ref. 2 that in certain materials, $l$ can be even

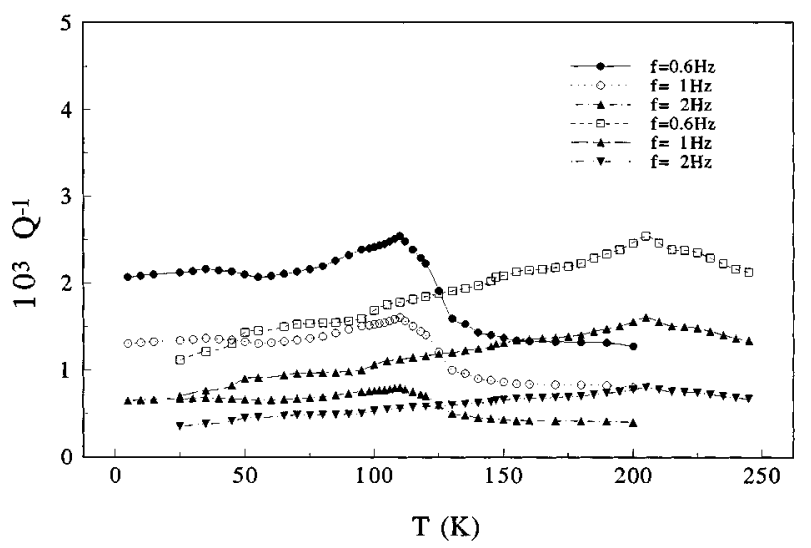

FIG. 9. Steady $Q^{-1}$ against temperature $T$. Note that each point here corresponds to a curve like that in Fig. 8. The black symbols pertain to various frequencies during cooling, while the white symbols pertain to different $f$ during heating, as marked.

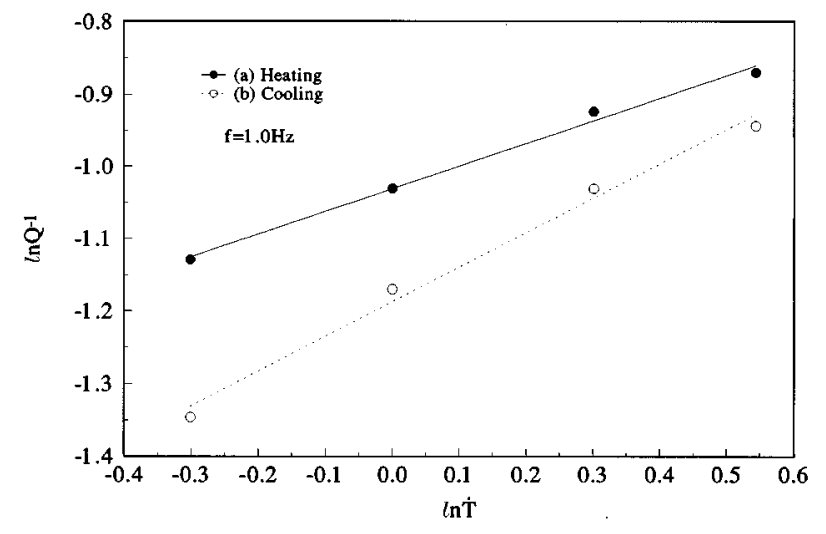

FIG. 10. $Q^{-1}$ against $\ln \dot{T}$ at $f=1.0 \mathrm{~Hz}$ during heating and cooling.

larger than $n$ and has its own physical meaning which is useful in characterization of the FOPT; $l$ should not be ignored.

In the previous sample $\left(\mathrm{VO}_{2}\right)$, a minimum and a clear dependence of $\mu$ on $\dot{T}$ and $f$ of the shear modulus occur, leading to the evaluation of the parameter $l$ through the plotting of $\left[Q_{p}^{-1} /(\Delta \mu / \mu)_{p}\right]$ vs $\dot{T}$ and vs $\omega$ (see Figs. 3 and 4 of Ref. 2). Since such a minimum does not appear here, neither is there a clear dependence of $\mu$ on $\dot{T}, f$, in the $\mu-T$ graph of this Fe-18.8\% Mn sample, we need to use Eq. (1) directly to find both values of $l$ and $n$ by a relatively complex numerical parametrization process. From Eq. (1), we have

$$
\left(Q^{-1} \omega^{2 l}-B \omega\right)=A(\dot{T} / \omega)^{n}
$$

or

$$
\ln \left(Q^{-1} \omega^{2 l}-B \omega\right)=n \ln (\dot{T} / \omega)+\ln A .
$$

For each value of $Q_{p}^{-1}$ and corresponding values of $\dot{T}$ and $\omega$, we take $l$ and $B$ as parameters and plot $\ln \left(Q_{p}^{-1} \omega^{2 l}-B \omega\right)$ against $\ln (\dot{T} / \omega)$, and calculate the root-mean-square error $\Delta \sigma$ of the points about the straight line. From the minimum of $\Delta \sigma$ or the maximum value of the standard linear correlation coefficient $R$, the values of $l, B, n$ (slope) and $\ln A$ (intercept) are therefore determined. We demonstrate in Fig.

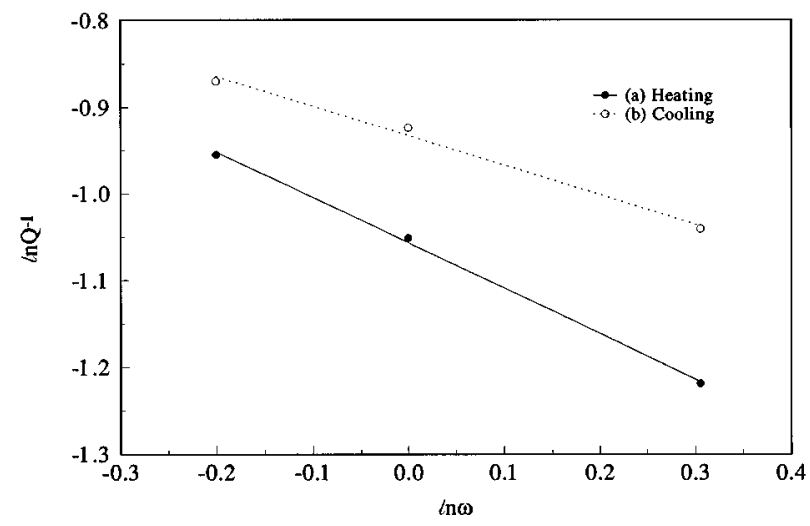

FIG. 11. $\ln Q_{p}^{-1}-\ln \omega$ relations during both heating and cooling (at constant $\dot{T}=2 \mathrm{~K} / \mathrm{min}$ ). 


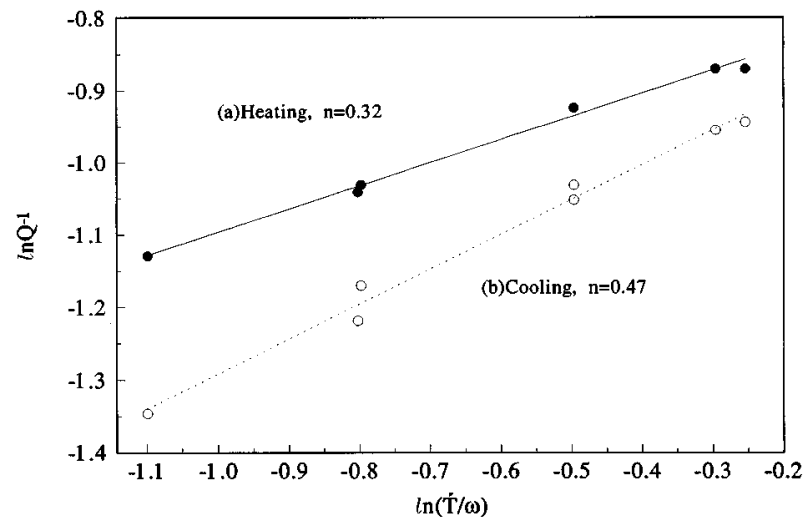
ing.

FIG. 12. $\ln Q_{p}^{-1}-\ln (\dot{T} / \omega)$ relations during both heating and cool-

13(a) the variation of $R$ and rms error $\Delta \delta$ with respect to change of the parameter $l$ for a series values of $B$ $\left(-2 \times 10^{-2}\right.$ to $2 \times 10^{-2}$ but the variation of $B$ values does not affect the values of $R$ and $\Delta \delta$ in this range) according to Eq. (6) during heating. Based on Fig. 13(a) alone, we accept that $l=0.015$ and $n=0.33$, corresponding to the minimum of rms error $\Delta \delta(<0.02)$. We show also in Fig. 13(b) the $R-l$ and $\Delta \sigma-l$ plots for a series values of $B$ (covering a range of $-2 \times 10^{-2}-2 \times 10^{-2}$ during cooling); the corresponding $\Delta \sigma$ values are indicated in the right vertical axis. We see that a clear minimum of $\Delta \delta(<0.03)$ occurs at $l=0.0035$ and $n=0.47$. Upon finding the parameters $l$ and
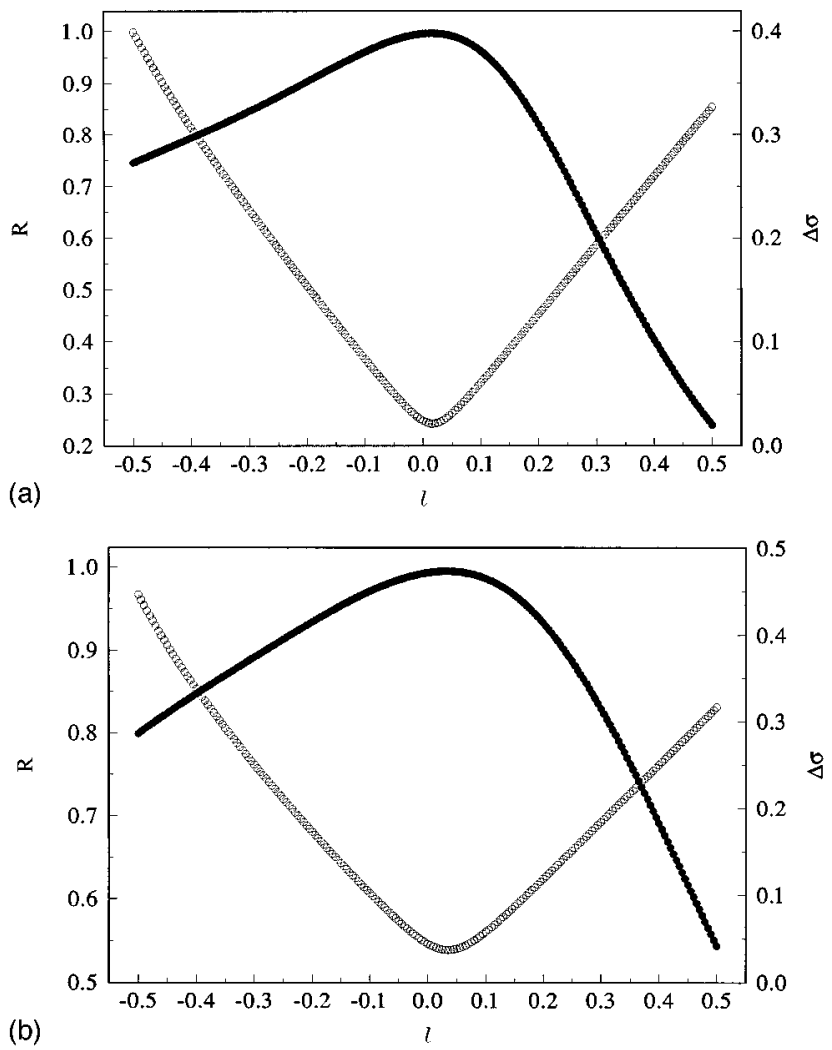

FIG. 13. (a) $R-l$ and $\Delta \delta-T$ plots during heating. (b) $R-l$ and $\Delta \delta-T$ plots during cooling.

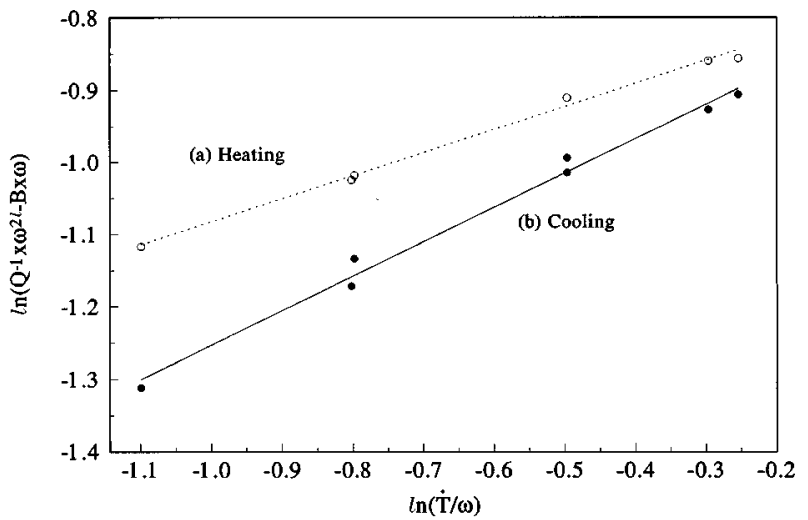

FIG. 14. $\left(\ln Q_{p}^{-1} / \omega^{1-2 l}-B \omega\right)$ vs $\ln (\dot{T} / \omega)$. (a) we find $n=0.33$, $l=0.015$, for $R>0.995$ during heating. (b) we find $n=0.47$, $l=0.035$, for $R>0.995$ during cooling.

$n$, we can now test Eq. (1) by plotting $\left(Q_{p}^{-1 /} \omega^{1-2 l}-B \omega\right)$ against $(\dot{T} / \omega)$ during both heating and cooling (Fig. 14). Since the value of parameter $B$ is at least three orders of magnitude smaller than that of parameter $A$ (see Fig. 9) and is in the range of the background value $\left(10^{-4}\right)$ of IF measurement, the value of $B$ is taken to be equal to the background value, i.e., $10^{-4}$ in Fig. 14.

Finally, from Fig. 9 we can measure the peak height for the static state by subtracting the background from the peak value $Q_{p}^{-1}$ of $Q^{-1}$. Such "static peak heights," i.e., $Q_{\mathrm{sp}}^{-1}$ occur at the same temperature $(383 \mathrm{~K})$ for three different frequencies $(0.6,1.0$, and $2.0 \mathrm{~Hz})$ during cooling. We plot in Fig. $15 \ln Q_{\mathrm{sp}}^{-1}-\ln f$ for that temperature. We repeat the $Q_{\mathrm{sp}}^{-1}$ measurement during the heating process and find that the three data points are practically identical to that for the cooling process, though occurring at a temperature of $478 \mathrm{~K}$ in this case.

For an isothermal process, the dynamic term of Eq. (1) is zero and the first term describes the static situation of internal friction, i.e.,

$$
Q_{\mathrm{sp}}^{-1}=B \omega^{1-2 l_{0}},
$$

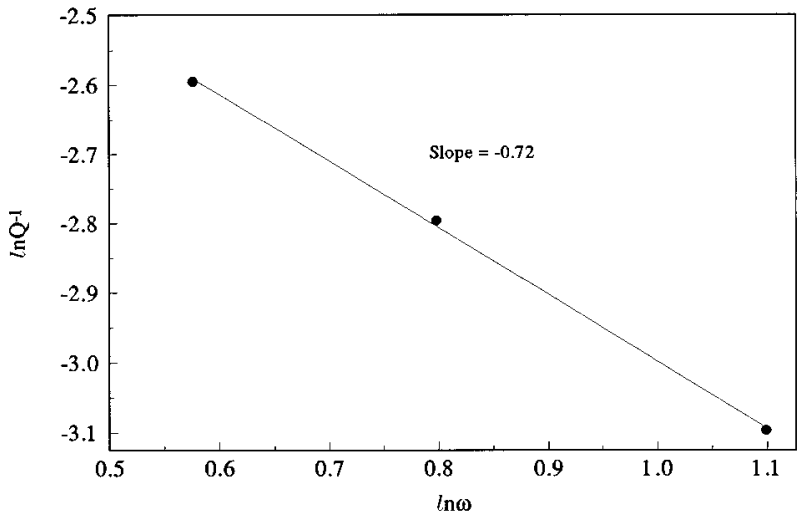

FIG. $15 . \ln Q_{\delta p}^{-1}$ vs $\ln \omega$ relation with slope $\left(1-2 l_{0}\right)=-0.72$ and $l_{0}=0.86$. 


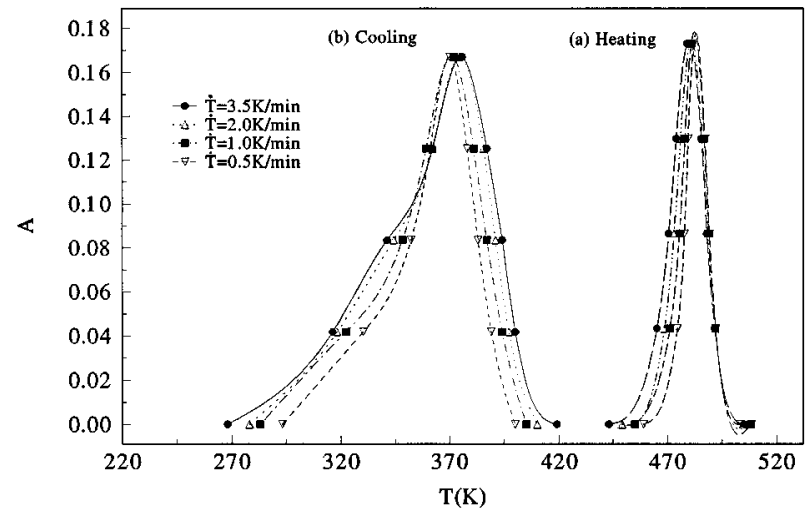

FIG. 16. $A(T)$ vs $T$ curves with different $\dot{T}$ as marked. (a) heating, (b) cooling.

here the symbol $l_{0}$ is used to replace the dynamic parameter $l$. The slope of Fig. 15 is found to be $\left(1-2 l_{0}\right)=-0.72$, giving $l_{0}=0.86$.

We now consider a $Q^{-1}-T$ curve for a certain $\dot{T}$. At a certain $T$, we can obtain a ratio $r=Q^{-1} / Q_{p}^{-1}$. For simplicity in analysis, we can take different values of $Q^{-1}$, corresponding to $r=1, \pm 3 / 4, \pm 1 / 2, \pm 1 / 4$, in the manner described in Ref. 2. We thus obtain a series of linear lines like those in Fig. 14, corresponding to different $r$ (or different $T$ ) values. The intercept is simply the function $A(T)$ in Eq. (6). We indicate the $A-T$ plot in Fig. 16 for different $\dot{T}$ during heating and cooling. We would remark that the temperature $T_{\max }^{c, h}$ (superscript $c$ stands for cooling and $h$ stands for heating) at which the maximum (of $A-T$ curve) occurs in a very narrow range, in contrast to the $\mathrm{VO}_{2}$ situation where $T_{\max }^{c}$ (or $T_{\max }^{h}$ ) shifts drastically as $\dot{T}$ changes. We also note that the whole $A-T$ curve shifts only slightly in this Fe-Mn sample, while that for the $\mathrm{VO}_{2}$ sample shifts and broadens significantly. The physical implications of these aspects will be treated later in this paper.

According to Eq. 2(a), it remains to find the parameter $k^{\prime}$ in order to determine the normalized dissipation function $\Delta G_{R}(T) /\left(\Delta H / T_{0}\right)$, as other parameters have been found. We have derived the explicit representation of $k^{\prime}$ in Eq. (8) of Ref. 2. Clearly we need to find $T_{v}$, which is the temperature at which $\Delta G_{R}$ is minimum (see Fig. 8 of Ref. 2). We first note that the rate of FOPT can be written as ${ }^{1}$

$$
d F / d t=N_{A} V,
$$

where $F$ is the volume fraction of the new phase, $N_{A}$ is the total area of the moving PI, and $V$ is the average velocity of the PI. For a FOPT with constant $\dot{T}$, we arrive at

$$
d F / d T=N_{A} V / \dot{T} .
$$

For a general FOPT, it is clear from Fig. 1 of Ref. 1 that the maximum of the $d F / d T-T$ curve and that of the $V-T$ curve occurs at the same temperature $T_{v}$. It is reasonable to assume that the maximum velocity of PI corresponds to the minimum of resistance, i.e., $\Delta G_{R}\left(T_{v}\right)$. Now the shear modulus is a function of the volume fraction of new phase $F$. In a relatively general term, we can write ${ }^{15}$

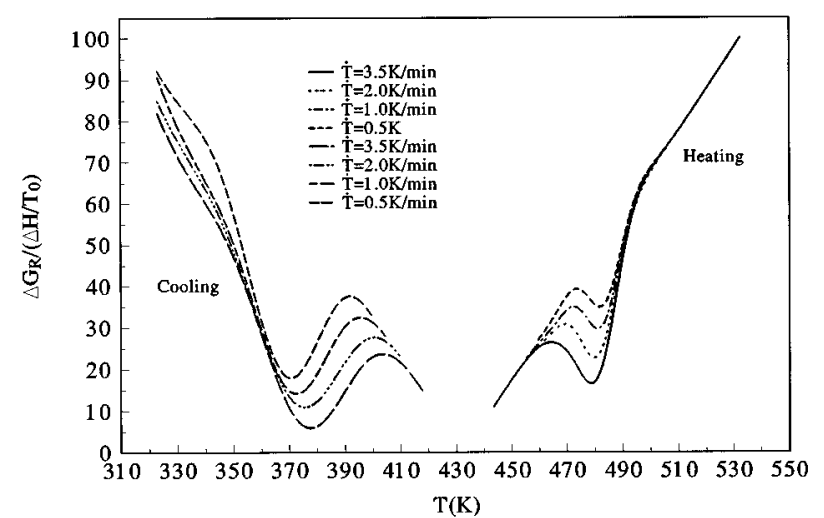

FIG. 17. Dissipation function $\Delta G_{R}(T)$ for FeMn alloy $\varepsilon / \gamma$ transformation with different $\dot{T}$ as marked.

$$
\mu=\mu_{M} F+\mu_{r}(1-F)
$$

giving

$$
\frac{d \mu}{d T}=\left(\mu_{M}-\mu_{r}\right) \frac{d F}{d T}
$$

where $\mu_{M}$ and $\mu_{r}$ are shear modulus of martensite and parent phases, respectively, and $F$ is the volume fraction of the $M$ phase, so that $d \mu / d T \propto d F / d T$. Thus finding the maximum of $d \mu / d T$ from our $\mu-T$ curve gives us the specific $T_{v}$ value at which $d F / d T$ is maximum. Substituting these $T_{v}$ values into Eq. (8) of Ref. 2, we obtain $k^{\prime}$ in Eq. (2a), and we can plot $\Delta G_{R} / \Delta H / T_{0}-T$ for both the heating and cooling processes for four different $\dot{T}$ as shown in Fig. 17. Based on our results reported in this paper, we observe the following relevant characteristics related to Fe-Mn FOPT: (i) According to our measurement shown in Fig. 4, there is as much as $0.5 \%$ variation in length during $\gamma \rightleftharpoons \varepsilon$ FOPT; such a variation in length is much greater than that encountered for most alloys and metals. We anticipate that there is a large strain energy involved in this FOPT, meaning that a prominent maximum of the resistance $\Delta G_{R}-T$ plot should occur, as observed in Fig. 17. (ii) The absolute value of $\Delta G_{R}$ is large and the difference between the maximum $\Delta G_{R}$ and the minimum of $\Delta G_{R}$ is also large in Fig. 17, while that for the $\mathrm{VO}_{2}$ sample (Fig. 8 of Ref. 2) is relatively small (because $\Delta H$ of $\mathrm{Fe}-\mathrm{Mn}$ is about one order of magnitude larger than that of $\mathrm{VO}_{2}$ ), implying that there is a relatively large effective driving force for the decreasing stage of the resistance $\Delta G_{R}$ and thus leading to a high velocity $V$ of PI. It is now easy to understand the reason for the occurrence of $\gamma \rightarrow \varepsilon$ burst-type FOPT in the Fe-Mn alloy. (iii) In the usual nomenclature of metallurgy, the starting temperature $M_{s}$ of martensite transformation during cooling decreases as $\dot{T}$ increases, while in the situation of FeMn, the $M_{s}$ decreases as $\dot{T}$ decreases (see Fig. 16). Such a feature which occurs only in burst-type MPT, is different from that occurring in FOPT of metals and alloys. This happening might be expected for a burst-type MPT from the viewpoint of energy storage and its abrupt release. (iv) It is interesting to note that $\Delta H$ for Fe-Mn is a function of $\dot{T}$ and $\Delta H$ increases as $\dot{T}$ increases (see Ref. 16). Taking into consideration such $\Delta H(\dot{T})$ depen- 


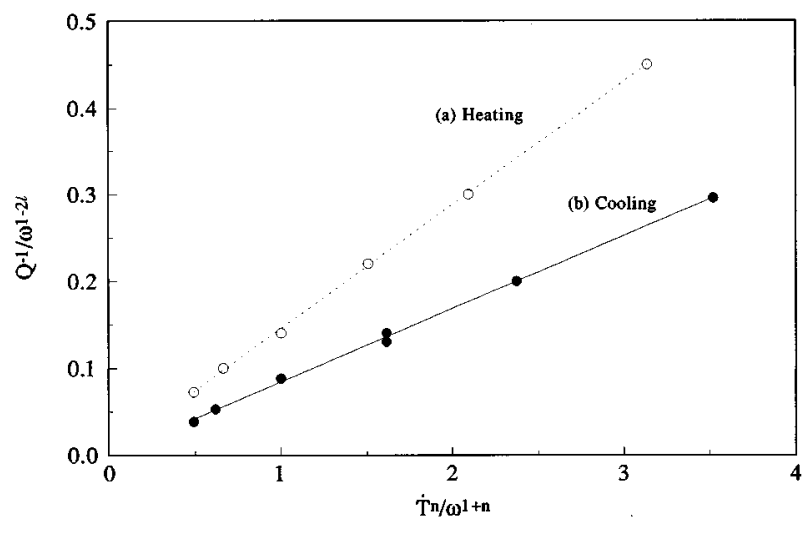

FIG. 18. $\left(Q_{\delta p}^{-1} / \omega^{1-2 l}\right)$ vs $\dot{T} / \omega^{1+n}$. (a) we find $n=0.59$, $l=0.06$ during heating. (b) we find $n=0.63, l=0.02$ during cooling.

dence, the $\Delta G_{R}$ value at high $\dot{T}$ should be higher than that at low $\dot{T}$. (v) For the temperature range $T<360 \mathrm{~K}$ in the cooling process, oscillation of the sample during internal friction measurement can induce a motion of the interphase between martensite domains (MDI). Part of the function $A(T)$ in Fig. 16 is contributed by such MDI, so that the $\Delta G_{R}-T$ plot below $360 \mathrm{~K}$ has qualitative meaning only.

\section{DISCUSSIONS AND CONCLUSIONS}

Comparing Figs. 6 and 7, the difference between $Q_{\delta}^{-1}$ and $Q_{w}^{-1}$ is large if $Q_{\delta}^{-1}>0.02$. Figure 18 shows the fitting results of Eq. (1) using $Q_{\delta}^{-1}$ (from Fig. 6) with $n=0.59, l=0.06$ for heating and $n=0.63, l=0.02$ for cooling, respectively. The values of $n$ and $l$ calculated from $Q_{w}^{-1}$ are listed in Table I.

Note, from Table I, the values of $n$ calculated from $Q_{\delta}^{-1}$ are around $70 \%$ larger than that from $Q_{w}^{-1}$. It is therefore necessary to use the data of $Q_{w}^{-1}$ for obtaining a realistic dependence of IF on $\dot{T}$ and $\omega$. In earlier works reported in Refs. 8 and 9 , because the value of $n$ was calculated from $Q_{\delta}^{-1}, n$ was overestimated.

Any acceptable theory should lead to consequences which are consistent with basic physical concept realizable in the phenomenon. Our theory of FOPT, though somewhat complex as it stands in Ref. 1, is built on a simple physical model in which a PI migrates during the phase transition. The special feature of our methodology is to introduce oscillations in the sample and measure the internal friction and shear modu- lus so that in the mathematical representation more equations and more unknowns are used to describe the dynamic system, leading to the situation where effectively the number of equations is equal to the number of unknowns. In the regime of the formulation, the parameters or unknowns which have definite physical meanings are $l, l_{0}, n, \Delta G_{R}$, and $\Delta G^{\prime}$, and we shall discuss their physical implications using the numerical values found in this investigation.

In the original formulation, $\Delta G_{d}$ is the driving force on the PI. There is a resistance force per unit area $\Delta G_{R}$ acting on the PI arising from three sources as stated in Ref. 1, so that the net or effective driving force on the PI is

$$
\Delta G^{\prime}=\Delta G_{d}-\Delta G_{R}=k^{\prime} \frac{A(T)}{B(T)}\left(T-T_{s}\right)^{n},
$$

where $k^{\prime}[A(T) / B(T)]$ describes the general shape, which is a bell shape of the $\Delta G^{\prime}-T$ curve. The power factor $\left(T-T_{s}\right)^{n}$, upon multiplying $k^{\prime}[A(T) / B(T)]$, does not change the bell shape nature of $A G^{\prime}$, but shifts the magnitude of $\Delta G^{\prime}$ and the $T_{p}$ value at which the peak occurs. We can thus interpret $A(T) / B(T)$ as the function specifying the shape of $\Delta G^{\prime}$. For the FeMn alloy, $\left(T_{p}-T_{s}\right) \sim 50$. The factor $\left(T-T_{s}\right)^{n}$ is 7.07 for $n=0.5$ and 1.48 for $n=0.1$, and the ratio 7.07/1.48 is about 5. Consequently, the magnitude of $\Delta G^{\prime}$ within the range $T_{f}-T_{s}$ is strongly dependent on $n$ according to Eq. (8) and we can take $n$ to be a measure of the net or effective driving force $\Delta G^{\prime}$ on PT. The parameter $n$ may be called the effective driving coefficient.

Introducing an internal friction measurement effectively sets up coupling between the external stress and the oscillations of PI. The application of the theory on the $\mathrm{VO}_{2}$ sample suggests that the coupling coefficient depends on the applied frequency $\omega$ in the simple manner $\alpha(\omega)=\alpha^{\prime} \omega^{l}$, as depicted in theory. Based on the $Q^{-1}(\omega)$ data and numerical analysis presented in Sec. IV of this paper, we have obtained the consequence that $l$ is non-negative (see Table I) for the $\mathrm{Fe}-\mathrm{Mn}$ sample. Hence we have a further test on the validity of the explicit expression $\alpha(\omega)=\omega^{-l}$. The value $l$ thus found can be tested for self-consistency together with other parameters. During our $Q^{-1}$ measurement, we kept the minimum at each constant temperature to ensure that the PI does not migrate. Thus when we substitute the condition $\dot{T}=0$ in Eq. (7), the internal friction measured, i.e., $Q^{-1}$ represents the static value and the coupling parameter deduced from the $Q^{-1}-\omega$ curves is the static one. In other words,

$$
Q_{s}^{-1}=B(T) \omega^{1-2 l_{0}}
$$

TABLE I. Values of $n$ and $l$ calculated from $Q_{\delta}^{-1}$ and $Q_{w}^{-1}$.

\begin{tabular}{lcccccccc}
\hline \hline & \multicolumn{4}{c}{$n$} & \multicolumn{2}{c}{ From $Q_{\delta}^{-1}$} & & \multicolumn{3}{c}{ From $Q_{w}^{-1}$} \\
& Heating & Cooling & Heating & Cooling & Heating & Cooling & Heating & Cooling \\
\hline$Q^{-1} \propto \dot{T}^{n}$ & 0.57 & 0.68 & 0.035 & 0.035 & 0.33 & 0.44 & 0.005 & 0.045 \\
$Q^{-1} \propto f^{-n}$ & 0.64 & 0.75 & 0.035 & 0.035 & 0.34 & 0.53 & 0.005 & 0.045 \\
$Q^{-1} \propto(\dot{T} / \omega)^{n}$ & 0.61 & 0.70 & 0 & 0 & 0.32 & 0.47 & 0 & 0 \\
Eq. (1), $\dot{T} \neq 0$ & 0.59 & 0.63 & 0.06 & 0.02 & 0.33 & 0.47 & 0.015 & 0.035 \\
Eq. (7), $\dot{T}=0$ & & & 0.86 & 0.86 & & & 0.86 & 0.86 \\
\hline \hline
\end{tabular}


under the condition $\dot{T}=0$. When $\dot{T} \neq 0$ and when the PI starts to migrate, the coupling parameter $l$ must take on the "dynamic value" which is in general significantly smaller than the static one. For example, according to Table I, $l=0.015$ (heating), 0.035 (cooling), while $l_{0}=0.86$ for this Fe-Mn sample. In the $\mathrm{VO}_{2}$ case, $l=0.2$ which $l_{0}$ is extremely large-there is no response of the PI to external stress. As a consequence, when the PI starts to move, $l_{0}$ does not exist and two terms have to be taken in Eq. (1) to describe $Q^{-1}$. The parameter $l$ or $l_{0}$ describes the degree of response of the external oscillating stress. In other words, $l$ or $l_{0}$ is small if the amplitude of the PI oscillation is large for a certain stress. We may call $l, l_{0}$ the stiffness parameters for the dynamic and static cases, respectively. We have found in this investigation that $l, l_{0}$ for the Fe-Mn sample are of the order of

*Present address: Lam Research Corp., CA 94583-6470.

†Present address: Dept. of Materials Science, Auburn University, Auburn, AL 36830.

${ }^{1}$ J. X. Zhang, P. C. W. Fung, and W. G. Zeng, Phys. Rev. B 52, 268 (1995).

${ }^{2}$ J. X. Zhang, Z. H. Yang, and P. C. W. Fung, Phys. Rev. B 52, 278 (1995).

${ }^{3} \mathrm{Z}$. Y. Xu, Martensitic Transformation and Martensite (Academic, Beijing, 1980), Chaps. 1 and 8.

${ }^{4}$ J. Spreadborough et al., Acta Crystallogr. 13, 603 (1960).

${ }^{5}$ A. Holdend et al., J. Iron Steel Inst. 209, 721 (1971).

${ }^{6}$ M. Tolba-Sallan et al., Scr. Metall. 15, 849 (1981).

${ }^{7}$ K. Shimizu, in Proceedings of Symposium on Martensite Trans- magnitude of $10^{-2}$ and $10^{-1}$, respectively. For the $\mathrm{VO}_{2}$ case, the dynamic coupling coefficient is about one order of magnitude higher, while the static one is extremely large as stated. So far, we have tested the theory with two types of FOPT. Further analyses with other specimens offering FOPT with different characteristics will be fruitful in understanding FOPT in solids.

\section{ACKNOWLEDGMENTS}

The work of J. X. Zhang, Y. Lin, and Z. C. Lin were supported by the Chinese National Science Foundation and Guangdong Province Natural Science Foundation. P.C.W. Fung is Visiting Professor to Zhongshan University, and is partly supported by RGC and CRCG grants in Hong Kong for this project. formation 82, edited by Z. Y. Xu (ASM, Shanghai, 1982), p. 44. ${ }^{8}$ Y. N. Wang and J. Z. Zhu, Acta Phys. Sin. 15, 341 (1959).

${ }^{9}$ Y. N. Wang et al., Acta Nanjing Univ. 7, 1 (1963).

${ }^{10}$ Y. L. Ma and T. S. Ke, Acta Phys. Sin. 20, 910 (1964).

${ }^{11}$ Z. J. Yang and Y. N. Wang, Acta Metall. Sin. 18, 21 (1982).

${ }^{12}$ H. M. Shen and Y. N. Wang, Acta Phys. Sin. 31, 1449 (1982).

${ }^{13}$ V. S. Postnikov et al., Nuovo Cimento B 33, 1125 (1976).

${ }^{14}$ U. Hiromich and Y. Hayakawa, J. Phys. Soc. Jpn. 21, 1281 (1966); 22, 205 (1967).

${ }^{15}$ L. E. Nielsen and R. F. Landel, Mechanical Properties of Polymers and Composites (Dekker, New York, 1994), Chap. 8.

${ }^{16}$ J. X. Zhang, F. Zhong, and G. G. Siu, Solid State Commun. 97, 847 (1996). 